\title{
Neuroscience with Music (5 September 2019, Berlin)
}

The first International Symposium on Neuroscience with Music was held at the beginning of September 2019 in Berlin. The meeting was established by Academus $\mathrm{GmbH}$, which organizes events within the scientific community as well as for civil society or government. This made the platform somewhat independent of academic institutions, thus the audience from the research fields of humanities and sciences was not the only one attracted by the symposium which interested at the same time delegates from various fields engaged in taking care of the soul and developing one's creative potential. The symposium also declared an effort to bridge art and science - this is indicated by the "with" in the title of the conference - which resulted in several artistic performances framing the scientific programme and in some cases also meant the active engagement of all the participants. These common experiences surely helped the atmosphere of sharing ideas.

The symposium's core topic was introduced by Vsevolod Shurhkhay, a neurosurgeon, who presented the basics and recent findings in the neurophysiological processing of sound and music.

A deeper view into the selected brain mechanisms of processing music was provided by Mendel Kaelen in his paper "Music as Psychedelic Medicine: Research Findings and Future Prospects." On the basis of his previous research into the brain mechanisms of psychedelic therapies, he considered the use of music in psychedelic research and therapy. Music is shown to be an effective tool in evoking emotions which can be employed in psychedelic-assisted psychotherapy. The possibilities of treatment of psychiatric disorders within this framework have been further approached and the technical apparatus developed for this purposes was presented together with the institutional support it gained.

This presentation foreshadowed the therapeutic approach provided by some other speakers, namely that of Andrei Anatoljevich Bogatyrev who presented the results of his research conducted together with Natalja Valerjevna Krasilnikova, focused on "Audio-Therapy and Rethinking Educational Guidelines for Doctor to Patient Communication based upon a Holistic Approach, Multimodal Semiotic Inspiration and Bioethics Principles". The next contribution centred on the therapeutic setting was presented by Yana Larionova with her paper 
"Music \& Psychotherapy: Two Ways to the Same Goal," led within the Gestaltist psychotherapeutic tradition.

The idea of modulating one's mind, especially emotions, resonated in the neuroscientific lecture of Stefan Ehrlich who spoke about "A Closed-loop, Musicbased Brain-computer Interface for Emotion Mediation". Ehrlich presented a non-invasive EEG-based brain-computer interface providing measurement of affective responses and affective neurofeedback, based on a two-dimensional model of affective space (arousal, valence). With the use of music stimuli automatically synthesised according to an algorithm, he registered participants' ratings of their emotional states to evaluate the match between intended and experienced emotion. He was then able to develop a musical feedback based on participants' emotional self-regulation: self-induced emotions were registered and led to a modification of automatically generated music. His paper clearly demonstrated the potential of therapeutic gains from technologies of brain research (software-based change of musical parametres, neurofeedback).

Technological tools were also presented by Alexander Lechner, coming from the commercial sphere of neurotechnology, where he is involved in developing EEG-based brain-computer interfaces, the function of which he presented to the audience with the aid of a volunteer and a musical component provided by Jan Slak and Hanna Preuss.

The symposium declared its aspiration to bridge art and science, not only to reflect but also to implement the interaction of mind and music, which found its fulfilment in several musical performances framing the scientific programme. The symposium began with an introductory organ performance by Ekaterina Melnikova who was also responsible for the conclusion of the entire event in the form of a recital, offering colourful arrangements of pieces from various genres, including popular music. Considerable contributions came also from Hanna Preuss and Jan Slak, who employed an improvisational approach in their flute and drummer performance ("The Sonorous Universe of Man: Presentation \& Performance"), Maria Kaplan (Aramaic Songs: Healing Resonance for the Regeneration of the Body), the English Choir Berlin with its conductor Kathleen Bird and Nanateya reproducing sounds from crystal bowls, a sort of musical meditation in the performance "Wave Art - Crystal Sound of the Universe". All the participants also engaged in short collective percussive performances under the guidance of Almut Lustig.

The second edition of the event, the format of which is to be announced, is planned for the following year. It may be also worthwhile to mention "The Neurosciences and Music-VII", a four-day conference that is going to be held in June 2020 in Aarhus, Denmark. 